\title{
Mathematical Approach to the Platonic Solid Structure of MS2 Particles
}

\author{
Nai-Chia Shih1, Chun-Chun Wu1 ${ }^{*}$, Jui-Sen Yang2 \\ ${ }^{1}$ Department of Applied Mathematics, Feng-Chia University, Taichung, Taiwan \\ ${ }^{2}$ Institute of Marine Biology, National Taiwan Ocean University, Keelung, Taiwan \\ Email: niga168@yahoo.com.tw, ${ }^{*}$ ccwu@math.fcu.edu.tw, jsyang@mail.ntou.edu.tw
}

Received 15 November 2014; accepted 10 April 2015; published 17 April 2015

Copyright (C) 2015 by authors and Scientific Research Publishing Inc.

This work is licensed under the Creative Commons Attribution International License (CC BY).

http://creativecommons.org/licenses/by/4.0/

(c) (i) Open Access

\begin{abstract}
Bacteriophage MS2 is a viral particle whose symmetrical capsid consists of 180 copies of asymmetrical coat proteins with triangulation number $T=3$. The mathematical theorems in this study show that the phage particles in three-dimension (3D) might be an icosahedron, a dodecahedron, or a pentakis dodecahedron. A particle with 180 coat protein subunits and $T=3$ requires some geometrical adaptations to form a stable regular polyhedron, such as an icosahedron or a dodecahedron. However, with mathematical reasons electron micrographs of the phage MS2 show that 180 coat proteins are packed in an icosahedron. The mathematical analysis of electron micrographs in this study may be a useful tool for surveying the platonic solid structure of a phage or virus particle before performing $3 \mathrm{D}$ reconstruction.
\end{abstract}

\section{Keywords}

MS2, Icosahedron, Mathematical Approach

\section{Introduction}

Many viruses and phages are icosahedral particles. However, spherical viruses may be polyhedral, forming dodecahedra or pentakis dodecahedra [1]. Bacteriophage MS2 is an icosahedral bacteriophage with a diameter of 27 - $34 \mathrm{~nm}$ [2] [3].

When an icosahedron is transferred to a flat sheet [4], its hexagon units consist of 6 regular triangles. A convex angle can be formed by 5 angles of regular triangles, but not 6 angles, which become a plane. The 20 triangular faces created by the vertices of 12 pentagonal cones and 30 edges can form an icosahedron.

The coat protein of MS2 forms a shell that protects the phage nucleic acid and acts as a translational repressor

"Corresponding author. 
[5]. The tertiary structure of the coat proteins is asymmetrical. A particle with nucleotides packed with asymmetrical proteins requires a low free-energy to achieve a stable condition. Packing as a helix or a regular polyhedron is a way of getting a symmetrical solid with asymmetrical subunits. In icosahedral particles, proteins are packed on the faces and directed to the vertices and the particles become symmetrical [3] [6]. In the MS2 capsid, one triangle of the icosahedron contains 3 asymmetrical subunits [6] [7]. This study uses mathematical analysis to identify the reasonable Platonic solids for packing a symmetrical capsid with asymmetrical subunits. Results show that the MS2 particles with 180 coat proteins and triangulation number $(T)=3$ might form an icosahedron, a dodecahedron, or a pentakis dodecahedron.

The overall shape of MS2 is spherical, but is difficult to see the three-dimensional (3D) figure in electron microscopy (EM) two-dimensional (2D) images before 3D reconstruction. Therefore, this study also introduces a mathematical method to predict particle solid 3D figures with 2D EM images before performing 3D reconstruction.

\section{Theory and Calculation}

The 3D structure of MS2 should be an isohedron or a regular polyhedron if the particles need to pack the asymmetrical proteins to make a stable symmetrical structure. The capsid of phage MS2 contains 180 identical copies of a coat protein with a $T=3$ isohedral (such as icosahedral) shell [3] [7]. MS2 particles may be constructed with 60 triangles, 45 quadrilaterals, 36 pentagons, or 30 hexagons. This study examines particle construction using the following theorems and mathematical analysis:

Theorem 1. If a polyhedron has 180 subunits, where 180/n-polygons have $n$ sides, $n$ should be a positive integer and equal or less than 5.

Proof. A polyhedron is constructed with $n$-side polygons, the polyhedron has $180 / n$ faces and the number of edges should be

$$
180 / n \times n \div 2=90 \text {. }
$$

By the Euler theorem, $V+F-E=2$, where $V, F$, and $E$ denote the number of vertices, faces, and edges, respectively,

$$
V=2+E-F=2+90-180 / n=92-180 / n .
$$

A vertex of solid angle needs at least trimer, then

$$
3 V \leq 180 \Rightarrow 3 \times(92-180 / n) \leq 180 \Rightarrow n \leq 5.625 .
$$

Thus, the maximum of $n$ is 5 , where the polygons are pentagons, quadrilaterals, or triangles.

Therefore, a polyhedron with 180 structure protein subunits should not be constructed with 30 hexagons.

Theorem 2. If a polyhedron with 180 subunits is constructed with 36 pentagons, the polyhedron should not be an isohedron or a regular polyhedron.

Proof. If 36 pentagons can construct an isohedron or a regular polyhedron, the number of faces is 36 . If two faces form dihedral angles, then the number of edges $=36 \times 5 \div 2=90$.

By the Euler theorem, $V+F-E=2 \Rightarrow$

$$
V=2+E-F=2+90-36=56
$$

If vertices are constructed with $x n$-polymers and $y$ m-polymers, where $x, y, n$, and $m$ are positive numbers, and $m>n$, then $n x+m y=180$.

Since $x+y=56$, that is,

$$
n x+n y=56 n \Rightarrow 56 n \leq 180 \Rightarrow n \leq 3.213
$$

the maximum of $n$ is 3 .

Thus, $3 x+m y=180$.

The shell of an isohedron or a regular polyhedron consists of $m$-polymer units, and $y$ m-polymers consist of 36 faces.

That is

$$
m y=36
$$

The solution of 3 simultaneous equations 


$$
\begin{gathered}
3 x+m y=180, \\
x+y=56, \\
m y=36,
\end{gathered}
$$

is $x=48, y=8, m=4.5$.

This is a contradiction with positive integer $m$.

Therefore, this theorem suggests that 36 pentagons cannot form an isohedron or a regular polyhedron.

Theorem 3. If a polyhedron with 180 subunits is constructed with an odd number of quadrilaterals, the polyhedron should not be an isohedron or a regular polyhedron.

Proof. Suppose that an isohedron or a regular polyhedron can be constructed with an odd number, $2 n+1$, of quadrilaterals.

The number of faces is then $2 n+1$, and the number of edges $=(2 n+1) \times 4 \div 2=4 n+2$.

By the Euler theorem, $V+F-E=2 \Rightarrow$

$$
V=2+E-F=2+4 n+2-2 n-1=2 n+3 .
$$

The number of vertices is odd, which is a contradiction because an isohedron or a regular polyhedron is symmetrical, and the number of verticies should be even.

Thus, an odd number (such as 45) of quadrilaterals cannot form an isohedron or a regular polyhedron.

According to Theorems 1, 2, and 3, 45 quadrilaterals, 36 pentagons, or 30 hexagons (the polygons with more than 3 edges) cannot form an isohedron or a regular polyhedron with 180 subunits. Only 60 triangles can form a regular polyhedron for packing 180 subunits.

Lemma 4. If a convex solid angle is constructed with $n$ equilateral triangles, $n$ should be 3 , 4, or 5 .

Proof. In 3D models, a convex solid angle has at least by 3 faces.

For a solid angle, the total angle with 3 equilateral triangular angles is $180^{\circ}$. The total angle with 4 equilateral triangular angles is $240^{\circ}$. The total angle with 5 equilateral triangular angles is $300^{\circ}$. The total angle with 6 equilateral triangular angles is $360^{\circ}$, which is a cyclic angle.

For a convex solid angle constructed with $n$ equilateral triangles, $n$ should be 3,4 , or 5 . The angle of a vertex equal to or greater than $360^{\circ}$ is flat or concave.

Theorem 5. If a polyhedron is constructed with 60 identical equilateral triangles, the polyhedron is not an isohedron or a regular polyhedron.

Proof. If a polyhedron is constructed with 60 identical equilateral triangles, as in Figure 1, then vertex Q in Figure 1 consists of 6 identical equilateral triangles.

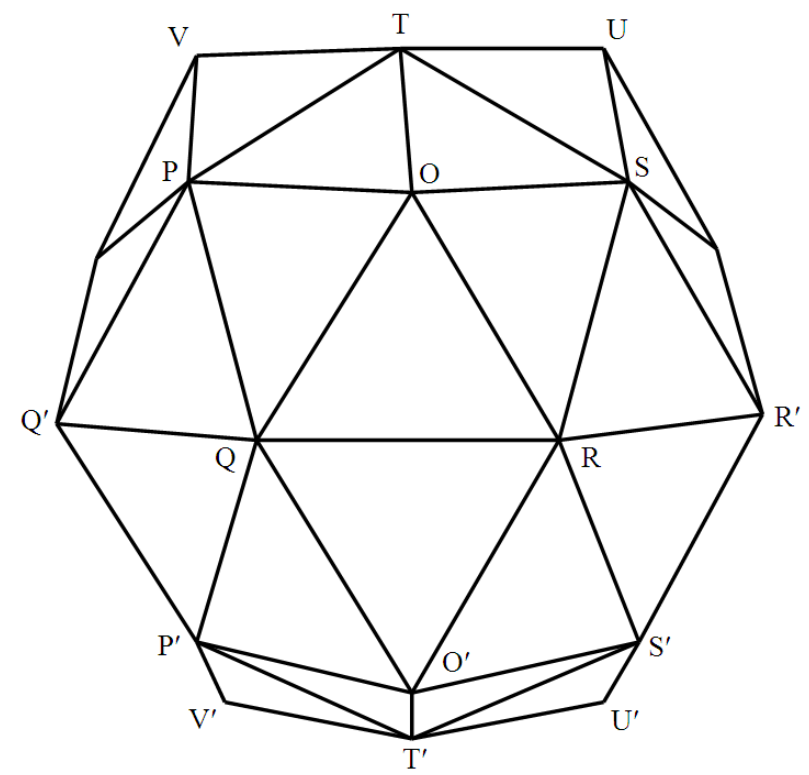

Figure 1. A convex polyhedron with 60 equilateral triangles. 
Lemma 4 shows that vertex $\mathrm{Q}$ is not a convex solid angle. Instead, vertex $\mathrm{Q}$ may belong to the following:

1) Vertex $Q$ is a concave solid angle.

This hypothesis is not true because the polyhedron is convex.

2) Point $Q$ is at the center of hexagon $P Q^{\prime} \mathrm{P}^{\prime} \mathrm{O}^{\prime} \mathrm{RO}$.

Vertex $\mathrm{O}$ and pentagon PQRST form a regular pentagonal cone. A dihedral angle forms between triangles $\mathrm{OPQ}$ and $\mathrm{OQR}$ on line $\overline{\mathrm{OQ}}$. Thus, polygon $\mathrm{PQ}^{\prime} \mathrm{P}^{\prime} \mathrm{O}^{\prime} \mathrm{RO}$ is not a regular hexagon, and the hypothesis is not true.

3) Vertex $Q$ locates on a dihedral angle.

Vertex $Q^{\prime}$ and pentagon $\mathrm{QP}^{\prime} \mathrm{T}^{\prime} \mathrm{S}^{\prime} \mathrm{R}$ form a regular pentagonal cone. Line $\overline{\mathrm{O}^{\prime} \mathrm{Q}}$ is on the dihedral angle between triangles $\mathrm{O}^{\prime} \mathrm{P}^{\prime} \mathrm{Q}$ and $\mathrm{O}^{\prime} \mathrm{QR}$. On the other hand, a dihedral angle forms between triangles $\mathrm{OPQ}$ and OQR on line $\overline{\mathrm{OQ}}$, as in (2). Therefore, two dihedral angles appear at vertex $\mathrm{Q}$, and the hypothesis is not true.

Theorem 6. A polyhedron becomes an icosahedron when the solid angles of the hexamers of the polyhedron spread to be flat and the connecting lines between the vertices of 2 nearby pentagonal cones become the ridges of dihedral angles.

Proof. As Figure 1 shows that the distances between the vertices of any 2 pentagonal cones in Figure 2 are the same. Then,

$$
\begin{gathered}
\overline{\mathrm{OO}^{\prime}}=\overline{\mathrm{OQ}^{\prime}}=\overline{\mathrm{OV}}=\overline{\mathrm{OU}}=\overline{\mathrm{OR}^{\prime}} \text { and } \\
\overline{\mathrm{OO}^{\prime}}=\overline{\mathrm{OQ}^{\prime}}=\overline{\mathrm{Q}^{\prime} \mathrm{V}}=\overline{\mathrm{VU}}=\overline{\mathrm{UR}^{\prime}}=\overline{\mathrm{R}^{\prime} \mathrm{O}^{\prime}}
\end{gathered}
$$

Spreading and flattening the solid angles of the hexamers in the pentagonal cone OO'Q'VUR' forms dihedral angles on lines $\overline{\mathrm{OO}^{\prime}}, \overline{\mathrm{OQ}^{\prime}}, \overline{\mathrm{OV}}, \overline{\mathrm{OU}}$, and $\overline{\mathrm{OR}^{\prime}}$. These lines become the edges of pentagonal cones, and vertex $\mathrm{O}$ has equilateral triangular faces. The vertices of 12 pentagonal cones have the same number of edges.

Therefore, a polyhedron becomes an icosahedron.

Theorem 7. A polyhedron becomes a dodecahedron when the solid angles of pentamers of the polyhedron spread out and become flat.

Proof. In Figure 3, for triangle OQR, triangle OQR becomes an isosceles triangle when lines $\overline{\mathrm{OQ}}$ and $\overline{\mathrm{OR}}$ decrease. Because

$$
\overline{\mathrm{OR}}<\overline{\mathrm{QR}} \Rightarrow \angle \mathrm{OQR}<\angle \mathrm{QOR}
$$



Figure 2. An icosahedron forms from a polyhedron after the solid angles of hexamers degenerate and the connecting lines (solid lines) between the vertices of 2 nearby pentagonal cones become the ridges of dihedral angles. 


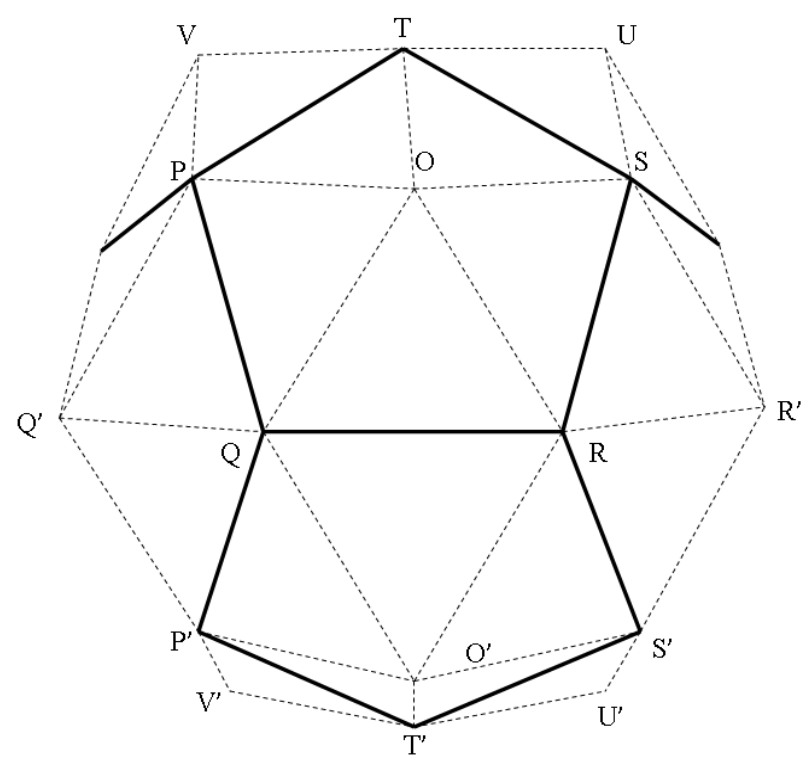

Figure 3. A dodecahedron forms from a polyhedron when the solid angles of pentamers (solid lines) spread out and become flat.

and

$$
\angle \mathrm{RQO}+\angle \mathrm{OQP}+\angle \mathrm{PQQ}^{\prime}+\angle \mathrm{Q}^{\prime} \mathrm{QP}^{\prime}+\angle \mathrm{P}^{\prime} \mathrm{QO}^{\prime}+\angle \mathrm{O}^{\prime} \mathrm{QR}<360^{\circ}
$$

Vertex Q becomes a convex solid angle and the polyhedron becomes a pentakis dodecahedron.

When the edge lines at vertex $\mathrm{O}$ decrease to allow the solid angle $\mathrm{O}$ to disappear and become flat, the pentagonal cone OPQRST flattens to become a pentagon PQRST.

Finally, the polyhedron becomes a dodecahedron.

\section{Results and Discussion}

\subsection{Mathematical Theorems in Packing a Stable Particle}

Theorems 1, 2, and 3 show that 45 quadrilaterals, 36 pentagons, or polygons with 6 or more edges cannot form an isohedron or a regular polyhedron with 180 subunits. Only 60 triangles can form a regular polyhedron for packing 180 subunits. In the MS2 particle, the way to pack 180 coat proteins is to form an isohedron or a regular polyhedron with 60 identical triangles (Lemma 4). The 3D polyhedron constructed with 60 identical equilateral triangles in Figure 1 is not an isohedron or a regular polyhedron (Theorem 5). That is, a polyhedron consisting of 60 identical equilateral triangles is not a convex isohedron. Therefore, a $T=3$ MS2 phage with 180 coat protein subunits might become an icosahedron [3] only if the solid angles of hexamers in the polyhedron of 60 identical equilateral triangles spread to become flat and the connecting lines between the vertices of 2 nearby pentagonal cones become the ridges of dihedral angles (Theorem 6). Theorem 7 shows that the MS2 particle model may form a dodecahedron when the solid angles of pentamers spread out and become flat.

The 3D structure of MS2 [3] consists of symmetrical units that lie between 2 threefold axes and 1 fivefold axis. However, a particle with 180 coat protein subunits and $T=3$ requires some geometrical adaptations to pack a stable regular polyhedron including an icosahedron or a dodecahedron (Theorem 6 and 7).

An array of hexamers is the basic unit for generating an icosahedron [4]. A hexagon consisting of 6 regular triangles cannot form a solid angle, but lies flat (Lemma 4). Removing a regular triangle from the triangles in the hexagon forms a pentagonal cone. The vertex of the pentagonal cone becomes one of the 12 solid angles of an icosahedron and the 20 triangular faces form part of hexagons [8].

For MS2 capsids with the principal of quasi-equivalence [8], the triangulation number $\left(T=h^{2}+h k+k^{2}\right)$ in the hexagon net is 3, where $h$ and $k$ are nonnegative integers on the original hexagonal net, $h$ and $k$ cannot be zero simultaneously, and the capsid has 180 structure subunit $(S)$ proteins $(S=60 T)$ and 32 morphological 
units $(M) \quad(M=10 T+2)$ [8]. $T$ values may be $1,3,4,7,9,12,13,16,19,21,25$, etc [1] although $T=2$ and 6 appear [9] [10]. When $T>1$, the morphological unit appears to form pentamers or hexamers. In an assembly pathway, 5 dimers converge into a pentamer. Twelve pentamers are linked together with free dimers creating a complete particle [2]. According to Theorems 6 and 7, a $T=3$ particle can form a regular polyhedron with geometrical degeneration. Although the numbers of subunit proteins vary, the particle morphology is quasi-equivalent.

This study hypothesizes that regular Platonic solids allow a single type of asymmetric subunit to assemble into a well-defined spherical structure [7]. The asymmetrical subunit contains 3 subunits, designated as A, B, and $\mathrm{C}$, in an icosahedral particle of phage MS2. Pairwise interactions between the monomers form dimers. The capsid contains 2 types of dimers: one at the quasi-twofold axis composed of subunits A and B and the other at the icosahedral twofold axis consisting of $2 \mathrm{C}$ subunits [7]. Therefore, the capsid is effectively constructed from 90 dimers [7]. The theorems above indicate that the $T=3$ phage MS2 particles with 180 protein subunits may by icosahedral, dodecahedral, or pentakis dodecahedral (dual semiregular solid) particles. Therefore, further studies with EM images are necessary to determine if the Platonic solid of MS2 is an icosahedron or a dodecahedron.

\subsection{Mathematical Reason with Electron Micrographs of MS2 Particles}

With electron micrographs [11], it is difficult to distinguish between regular hexagons and hexagon-like dodecagons in the projections of icosahedron. Both regular hexagons and regular decagons appear in EM images, though regular decagons appear spherical (Figure 4). However, unequilateral hexagons with large obtuse angles instead of narrow obtuse angles appear in EM images of MS2 in twofold views (Figure 5).

The 5 regular Platonic solids are tetrahedron, octahedron, cube, dodecahedron, and icosahedron. The icosahedron has a common symmetry with the dodecahedron, and the octahedron is similar to the cube [7]. Most studies recognize the bacteriophage MS2 as an icosahedral particle [1]-[3] [6] [12] [13], though the MS2 coat protein mutant corresponds to $T=3$ octahedral particles [9]. The main difference in the subunit packing between the octahedral and icosahedral arrangements is close to the fourfold and fivefold symmetry axes [7].

The mathematical reason from Theorem 6 and 7 shows that the $T=3$ phage MS2 particles may be icosahedral, dodecahedral, or pentakis dodecahedral particles. The projections of icosahedral particles in phage MS2 EM images exhibit unequilateral hexagons in twofold views, regular hexagons in threefold views, and regular decagons in fivefold views (Figure 4). The projections of dodecahedron are unequilateral hexagons in twofold views, hexagon-like dodecagons in threefold views, and regular decagons in fivefold views and asymmetrical views. The projections of pentakis dodecahedra are regular decagons in twofold, threefold, and fivefold views (Table 1). In fivefold views, icosahedra, dodecahedra, and pentakis dodecahedra models show the same projection as a

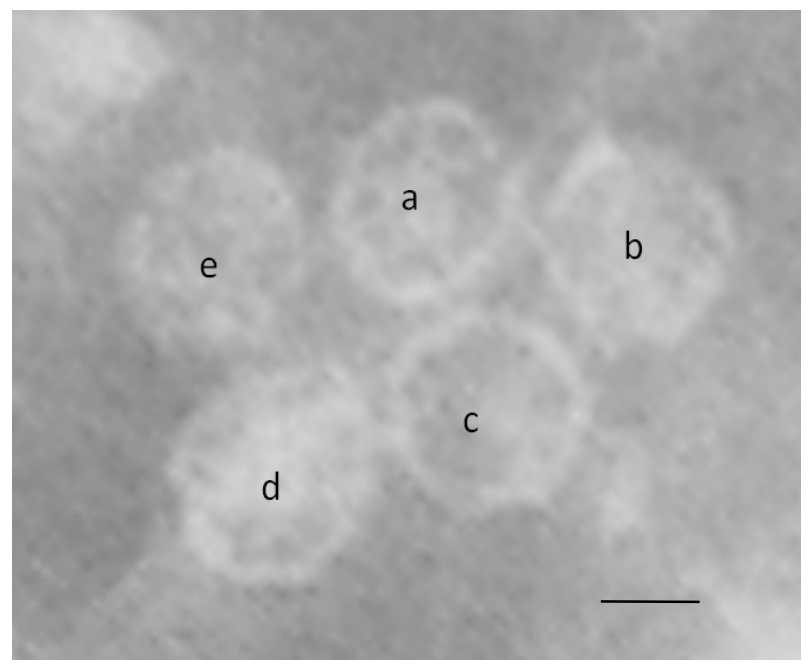

Figure 4. Micrograph of phage MS2 in twofold (a), threefold (b) and fivefold (c) views. Note particles attaching (d) and unattaching (e) on a pilus of E. coli. The bar represents 10 $\mathrm{nm}$. 
regular decagon. In threefold views, 3 models have different projections: regular hexagons form from icosahedra, hexagon-like dodecagons form from dodecahedra, and regular decagons form from pentakis dodecahedra. In twofold views, the projections of icosahedra and dodecahedra are unequilateral hexagons (Figure 6). In the unequilateral hexagons, 4 vertices have the same angles, and are unlike the other 2 obtuse angles. The 2 big obtuse angles in the unequilateral hexagons of the projections from icosahedra and dodecahedra are $138^{\circ}$ and $116^{\circ}$, respectively [14]. The obtuse angles of unequilateral hexagon projections from icosahedra are much larger than those from dodecahedra. EM images of the MS2 particles show the projections of icosahedral particles since the obtuse angles of unequilateral hexagons are $138^{\circ}$. The $T=3$ phage MS2 particles with 180 coat protein subunits [3] form icosahedra. This suggests that the solid angles of hexamers in the polyhedral particles may spread to become flat, and the connecting lines between the vertices of 2 nearby pentagonal cones become the ridges of dihedral angles during packing the coat protein subunits in MS2 (Theorem 6 and 7). A few viral particles are dodecahedra [4] [15]. In nature, spreading in the solid angles of hexamers in a polyhedron seems to be easier and more common than spreading of pentamers. Most viruses and phages form icosahedral particles instead of dodecahedral or pentakis dodecahedral particles.

\subsection{Mathematical Analysis in Particle Electron Micrographs for 3D Reconstruction}

Although the 3D structure reconstructed from the EM images shows the Platonic solids of the particles, the wrong order of 3D reconstruction might yield a false solid figure [16]. It is easy to recognize an icosahedron or a dodecahedron in the Platonic solid of a particle using EM images and mathematical analysis. According to a

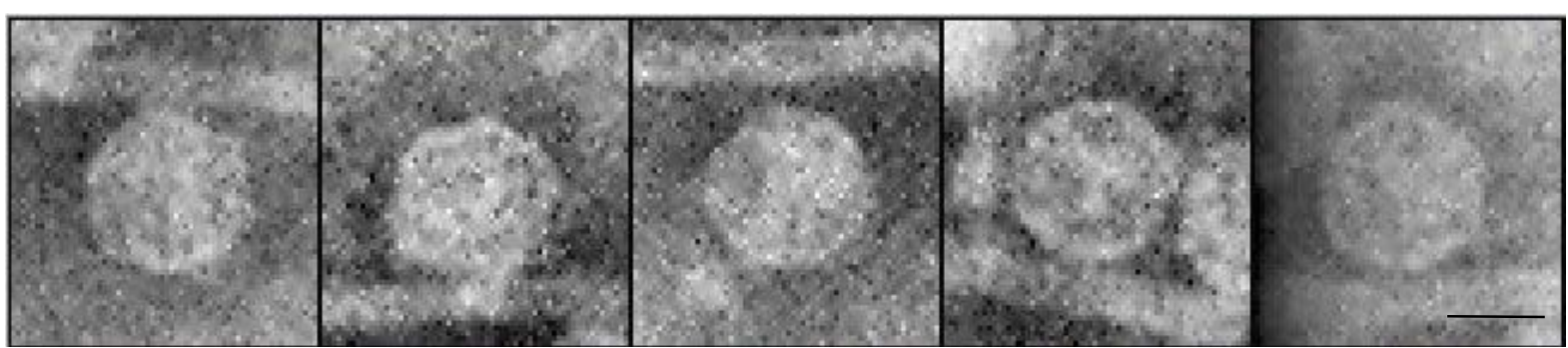

Figure 5. Micrographs of phage MS2 from twofold views. The bar represents $20 \mathrm{~nm}$.

Table 1. Projections of polyhedra from various views.

\begin{tabular}{cccc}
\hline & Twofold & Symmetry & Asymmetry \\
\cline { 2 - 4 } & Unequilateral hexagon & Regular hexagon & Regular decagon \\
Icosahedron & Unequilateral hexagon & Hexagon-like dodecagon & Regular decagon \\
Dodecahedron & regular decagon & Regular decagon & Regular decagon \\
\hline
\end{tabular}

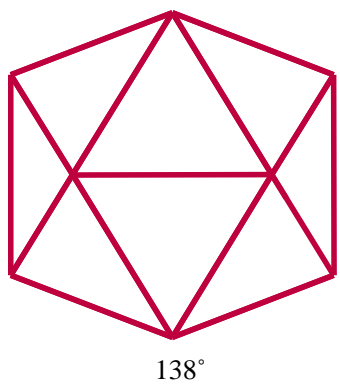

(a)

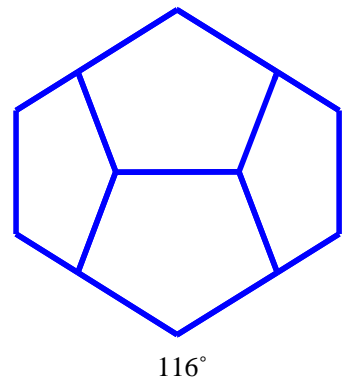

(b)

Figure 6. Projections of an icosahedron (a) and a dodecahedron (b) from a twofold view. 
primary survey of the Platonic solid of the particle, the 3D reconstruction can be performed confidently with a right symmetrical order [10] [14]. The mathematical analysis of EM images is a useful primary survey before performing the 3D reconstruction of a polyhedral particle.

\section{Conclusion}

In conclusion, a viral particle with 180 coat protein subunits and $T=3$ requires some geometrical adaptation to form a stable regular polyhedron, such as an icosahedron or a dodecahedron. The mathematical analysis of MS2 particles reveals the EM projections of icosahedral particles. The MS2 particles are confirmed to be icosahedra.

\section{Acknowledgements}

We gratefully acknowledge Mr. Jaw-Fu Gau for his EM assistance.

\section{References}

[1] Janner, A. (2006) Towards a Classification of Icosahedral Viruses in Terms of Indexed Polyhedral. Acta Crystallographica, Section A, 62, 319-330.

[2] Golmohammadi, R., Valegård, K., Fridborg, K. and Liljas, L. (1993) The Refined Structure of Bacteriophage MS2 at 2.8 Å Resolution. Journal of Molecular Biology, 234, 620-639.

[3] Valegård, K., Liljas, L., Fridborg, K. and Unge, T. (1990) The Three-Dimensional Structure of the Bacterial Virus MS2. Nature, 345, 36-41. http://dx.doi.org/10.1038/345036a0

[4] Baker, T.S., Olson, N.H. and Fuller, S.D. (1999) Adding the Third Dimension to Virus Life Cycles: Three-Dimensional Reconstruction of Icosahedral Viruses from Cryo-Electron Micrographs. Microbiology and Molecular Biology Reviews, 63, 862-922.

[5] Valegård, K., Murray, J.B., Stonehouse, N.J., van den Worm, S., Stockley, P.G. and Liljas, L. (1997) The Three-Dimensional Structures of Two Complexes between Recombinant MS2 Capsids and RNA Operator Fragments Reveal Sequence-Specific Protein-RNA Interactions. Journal of Molecular Biology, 270, 724-738.

[6] Koning, R., van den Worm, S., Plaisier, J.R., van Duin, J., Abrahams, J.P. and Koerten, H. (2003) Visualization by CryoElectron Microscopy of Genomic RNA That Binds to the Protein Capsid inside Bacteriophage MS2. Journal of Molecular Biology, 332, 415-422.

[7] Plevka, P., Tars, K. and Liljas, L. (2008) Crystal Packing of a Bacteriophage MS2 Coat Protein Mutant Corresponds to Octahedral Particles. Protein Science, 17, 1731-1739. http://dx.doi.org/10.1110/ps.036905.108

[8] Caspar, D.L.D. and Klug, A. (1962) Physical Principles in the Construction of Regular Viruses. Cold Spring Harbor Symposia on Quantitative Biology, 27, 1-24.

[9] Flint, S.J., Enquist, L.W., Krug, R.M., Racaniello, V.R. and Skaika, A.M. (2000) Principles of Virology: Molecular Biology, Pathogenesis, and Control. American Society for Microbiology Press, Washington DC, 58-98.

[10] Reddy, V.S. and Johnson, J.E. (2005) Structure-Derived Insights into Assembly. Advances in Virus Research, 64, 4568.

[11] Gau, J.F. (2010) The Attachment of MS2 on E. coli. Master thesis, National Taiwan Ocean University, Keelung.

[12] Plevka, P., Tars, K. and Liljas, L. (2009) Structure and Stability of Icosahedral Particles of a Covalent Coat Protein Dimer of Bacteriophage MS2. Protein Science, 18, 1653-1661. http://dx.doi.org/10.1002/pro.184

[13] Schneemann, A. (2006) The Structural and Functional Role of RNA in Icosahedral Virus Assembly. Annual Review of Microbiology, 60, 51-67. http://dx.doi.org/10.1146/annurev.micro.60.080805.142304

[14] Dr. Math, FAQ (1994-2009) (2009) Regular Polyhedral: Formulas. The Math Forum@Drexel. Drexel University, Philadelphia. http://mathforum.org

[15] Cann, A.J., (Au \& Ed) (2005) The Principles of Molecular Virology. Chap. 2, Particles. Elsevier Academic Press, San Diego, 25-55.

[16] Shih, N.C. (2009) Mathematical Studies on the 3D Reconstruction of Phage MS2. Master Thesis, Feng-Chia University, Taichung. 